\title{
Center for Epidemiologic Studies Depression Scale
}

National Cancer Institute

\section{Source}

National Cancer Institute. Center for Epidemiologic Studies Depression Scale. NCI Thesaurus. Code C77964.

A self-test that measures depressive feelings and behaviors within the general population, 